\title{
Optimizing gentamicin conventional and extended interval dosing in neonates using Monte Carlo simulation - a retrospective study
}

Monique Bergenwall ${ }^{1,8+}$, Sandra A. N. Walker ${ }^{1,2,3,4^{*}+}$ (D) Marion Elligsen ${ }^{1}$, Dolores C. laboni ${ }^{5}$, Carla Findlater ${ }^{5}$, Winnie Seto ${ }^{2,6}$ and Eugene $\mathrm{Ng}^{5,7}$

\begin{abstract}
Background: Although aminoglycosides are routinely used in neonates, controversy exists regarding empiric dosing regimens. The objectives were to determine gentamicin pharmacokinetics in neonates, and develop initial mg/kg dosing recommendations that optimized target peak and trough concentration attainment for conventional and extended-interval dosing (EID) regimens.

Methods: Patient demographics and steady-state gentamicin concentration data were retrospectively collected for 60 neonates with no renal impairment admitted to a level III neonatal intensive care unit. Mean pharmacokinetics were calculated and multiple linear regression was performed to determine significant covariates of clearance $(L / h)$ and volume of distribution (L). Classification and regression tree (CART) analysis identified breakpoints for significant covariates. Monte Carlo Simulation (MCS) was used to determine optimal dosing recommendations for each CARTidentified sub-group.

Results: Gentamicin clearance and volume of distribution were significantly associated with weight at gentamicin initiation. CART-identified breakpoints for weight at gentamicin initiation were: $\leq 850 \mathrm{~g}, 851-1200 \mathrm{~g}$, and > $1200 \mathrm{~g}$. MCS identified that a conventional dose of gentamicin $3.5 \mathrm{mg} / \mathrm{kg}$ given every $48 \mathrm{~h}$ or an EID of $8-9 \mathrm{mg} / \mathrm{kg}$ administered every $72 \mathrm{~h}$ in neonates weighing $\leq 850 \mathrm{~g}$, and every 24 and $48 \mathrm{~h}$, respectively, in neonates weighing 851-1200 g, provided the best probability of attaining conventional (peak: $5-10 \mathrm{mg} / \mathrm{L}$ and trough: $\leq 2 \mathrm{mg} / \mathrm{L}$ ) and EID targets (peak:12-20 mg/L, trough: $\leq 0.5 \mathrm{mg} / \mathrm{L}$ ). Insufficient sample size in the $>1200 \mathrm{~g}$ neonatal group precluded further investigation of this weight category.

Conclusions: This study provides initial gentamicin dosing recommendations that optimize target attainment for conventional and EID regimens in neonates weighing $\leq 1200 \mathrm{~g}$. Prospective validation and empiric dose optimization for neonates $>1200 \mathrm{~g}$ is needed.
\end{abstract}

Keywords: Neonate, Gentamicin, Pharmacokinetics, Traditional dosing, extended-interval dosing, Monte Carlo simulation

\footnotetext{
* Correspondence: sandra.walker@sunnybrook.ca

${ }^{\dagger}$ Monique Bergenwall and Sandra A. N. Walker contributed equally to this work.

'Department of Pharmacy, Sunnybrook Health Sciences Centre, 2075

Bayview Avenue, E-302, Toronto, ON M4N 3M5, Canada

${ }^{2}$ Leslie L. Dan Faculty of Pharmacy, University of Toronto, Toronto, ON,

Canada

Full list of author information is available at the end of the article
}

(c) The Author(s). 2019 Open Access This article is distributed under the terms of the Creative Commons Attribution 4.0 International License (http://creativecommons.org/licenses/by/4.0/), which permits unrestricted use, distribution, and reproduction in any medium, provided you give appropriate credit to the original author(s) and the source, provide a link to the Creative Commons license, and indicate if changes were made. The Creative Commons Public Domain Dedication waiver (http://creativecommons.org/publicdomain/zero/1.0/) applies to the data made available in this article, unless otherwise stated. 


\section{Background}

Although aminoglycosides are routinely used in neonates, controversy exists regarding recommended empiric dosing to optimize target attainment with either conventional dosing (peak: $5-10 \mathrm{mg} / \mathrm{L}$ and troughs $\leq 2$ $\mathrm{mg} / \mathrm{L}$ ) or extended-interval dosing (higher peak and undetectable trough) $[1,2]$. In adult and older pediatric populations, EID regimens targeting peak concentrations of $\geq 20 \mathrm{mg} / \mathrm{L}$ are routinely recommended based on data suggesting that aminoglycoside activity is optimized with peak: minimum inhibitory concentration (MIC) ratios of 8-10:1 [3-5]. For these patient populations, EID has consistently demonstrated equal efficacy, and equal or reduced toxicity versus conventional dosing $[1,6-10]$.

While data exist to support the use of EID in neonates $[6,11-26]$, consensus is lacking regarding optimal EID target concentrations that optimize efficacy and minimize toxicity in this patient population. Peak concentrations investigated in neonates vary from 4 to 20 $\mathrm{mg} / \mathrm{L}$ [15-26], and typically remain below $12 \mathrm{mg} / \mathrm{L}$, with no clear rationale. Furthermore, infants born at a gestational age $(\mathrm{GA}) \leq 28$ weeks, along with those with a birth weight (BW) of $\leq 1500 \mathrm{~g}$, are underrepresented in EID studies. These infants constitute approximately $20 \%$ of all neonates admitted to Canadian neonatal intensive care units (NICUs), and $50 \%$ of those admitted to Level III NICUs [27]. Since aminoglycoside pharmacokinetic (PK) parameters in neonates may be influenced by weight $[15,18-20,28,29]$, gestational age $[15,28,29]$ and postnatal age $[19,28,29]$, further research is required in this unique population in order to optimize target attainment and thereby, maximize the probability of efficacy of the antibiotic while minimizing the risk of nephrotoxicity.

The objectives of this study were to determine the pharmacokinetics of gentamicin in neonates with no clinical evidence of renal impairment in a Level III NICU, identify significant covariates of gentamicin PK parameters in neonates, and develop practical initial dosing recommendations with the highest probability of attaining target peak and trough serum concentrations currently accepted in clinical practice for both conventional dosing (trough $<2 \mathrm{mg} / \mathrm{L}$ and peak $5-10$ ) and EID (trough $<0.5 \mathrm{mg} / \mathrm{L}$ and peak $8-20 \mathrm{mg} / \mathrm{L}, 12-20 \mathrm{mg} / \mathrm{L}, 15-20 \mathrm{mg} / \mathrm{L}$ and $>20 \mathrm{mg} / \mathrm{L}$ ) of gentamicin.

\section{Methods}

This retrospective study was conducted in the level III NICU at Sunnybrook Health Sciences Centre (SHSC) in Toronto, Ontario, Canada. SHSC is a 1325-bed tertiary care teaching hospital, with 48 NICU beds [30].

\section{Patient eligibility}

Neonates admitted to the NICU from March 12th, 2010-November 26th, 2013 who were prescribed gentamicin to treat a documented or presumed infection and received $>48 \mathrm{~h}$ of gentamicin were identified from a hospital electronic database [31]. Patients with at least one set of steady state gentamicin serum concentrations (trough and peak concentrations obtained at the earliest before and after the third dose of a given dosing regimen, respectively) with documentation of gentamicin administration and serum sampling times were included.

Neonates were excluded if they developed acute renal failure (urine output $<1 \mathrm{~mL} / \mathrm{kg} / \mathrm{hr}$. or serum creatinine $[\mathrm{sCr}]>100 \mu \mathrm{mol} / \mathrm{L}$ ) before or during gentamicin therapy, had an increase in $\mathrm{sCr}>25 \%$ from baseline during treatment, or had a calculated gentamicin half-life > two standard deviations (SDs) from the mean half-life observed in the study population following data analysis, without the availability of an additional set of serum concentrations to confirm the accuracy of this calculated half-life.

\section{Gentamicin dosing and sampling procedure}

At the time of this study, neonatal SHSC conventional gentamicin dosing recommendations aimed to target a peak and trough serum concentration of $5-10 \mathrm{mg} / \mathrm{L}$ and $\leq 2 \mathrm{mg} / \mathrm{L}$, respectively. (Appendix 1).

\section{Gentamicin pharmacokinetics}

The PK profile of gentamicin in neonates has been previously described using one $[14,15,17-19,21$, 24], two [20, 25, 29] and three [28] compartment models. Once gentamicin distribution is complete, it follows first order elimination [15, 20, 28]. Therefore, a one compartment model is appropriate to evaluate the post-distribution pharmacokinetics of gentamicin. Gentamicin concentrations were analyzed using first order PK principles to calculate extrapolated gentamicin trough and peak, elimination rate constant $\left(\mathrm{k}_{\mathrm{e}}\right)$, half-life $\left(t_{1 / 2}\right)$, volume of distribution $(\mathrm{Vd})$, clearance $(\mathrm{Cl})$, initial estimated dose $(\mathrm{mg} / \mathrm{kg}$, rounded to nearest $0.5 \mathrm{mg}$ ) and dosing interval for conventional (trough $\leq 2 \mathrm{mg} / \mathrm{L}$ and peak $5-10 \mathrm{mg} / \mathrm{L}$ ) and EID (trough $\leq 0.5$ $\mathrm{mg} / \mathrm{L}$ and peak $8-20 \mathrm{mg} / \mathrm{L}, 12-20 \mathrm{mg} / \mathrm{L}, 15-20 \mathrm{mg} / \mathrm{L}$ and $>20 \mathrm{mg} / \mathrm{L}$ ) using an infusion time of $1 \mathrm{~h}$. (Appendix 2) When multiple sets of gentamicin serum concentrations were obtained from the same patient, each set was evaluated independently for inclusion, and if eligible, was included as a separate sample for the PK analysis along with the corresponding postnatal age (PNA) and corrected GA (CGA) at time of 
gentamicin initiation; weight closest to gentamicin initiation; and weight within $24 \mathrm{~h}$ of gentamicin levels.

\section{Microbiological cultures}

Data for all positive bacterial isolates along with the culture source were extracted from the hospital electronic data base and patient charts.

\section{Statistical analysis}

Descriptive statistics were used for patient characteristics and microbiological results (number, percent, mean, SD and range). Since PK parameters display a lognormal distribution, the geometric mean, 95\% confidence interval $(\mathrm{CI})$ and range were reported for $\mathrm{k}_{\mathrm{e}}$, $\mathrm{t}_{1 / 2}, \mathrm{Vd}$, and $\mathrm{Cl}$.

The data consisted of 60 neonates, of which only 4 had a second set of data with gentamicin levels. This sample size, along with the limited number of repeated measures, was insufficient to run a robust hierarchical model. To circumvent this problem, only data from the first set of gentamicin levels were included for the analyses. Clinical parameters that would have been known prior to the initiation of gentamicin, were not calculated using other parameters input into the regression analysis and were parameters with values available for $>80 \%$ of the gentamicin levels (GA at birth; CGA at gentamicin initiation; PNA at gentamicin initiation; gender; BW; weight at gentamicin initiation; Apgar score at one and $5 \mathrm{~min}$ of age; blood urea nitrogen [BUN] closest to gentamicin initiation, $\mathrm{sCr}$ closest to gentamicin initiation, $24 \mathrm{~h}$ urine output $[\mathrm{ml} / \mathrm{hr}]$, and albumin closest to gentamicin initiation; use of concomitant nephrotoxins [indomethacin, ibuprofen, furosemide, amphotericin B, vancomycin]; and small-for-gestational age [SGA; i.e neonates with a birth weight below the 10th percentile for neonates of the same GA] status) were input in the regression analysis. Variables that were significant $(p<0.05)$ with bivariate analysis and had a tolerance statistic of $\geq 0.4$ when assessed for multicollinearity were included in a multivariable linear regression (MLR) model to identify those that remained significant using a $p<0.05$. Analyses were run using SAS Version 9.4 (SAS Institute, Cary, NC, USA).

A Classification and Regression Tree (CART) analysis (CART1 Professional Extended Edition, Salford Systems, San Diego, California) was used to identify whether practical breakpoints existed for statistically significant MLR-identified covariates of gentamicin $\mathrm{Cl}(\mathrm{L} / \mathrm{h})$ and $\mathrm{Vd}(\mathrm{L})$. The initial CART analyses input all statistically significant variables identified in the MLR analyses for Vd (L) and/or Cl (L/h). CART analyses for $\mathrm{Cl}$ and $\mathrm{Vd}$ were pruned to the simplest tree, utilizing forced splits to identify clinically practical breakpoints, with the lowest relative error. Forced splits were selected as practical rounded breakpoints derived from the CART identified breakpoint and which had equal or lower relative error than the CART identified breakpoint. The optimal CART model was that which allowed for the fewest sub-groups and had the lowest relative error. CARTidentified breakpoints for covariates of gentamicin $\mathrm{Vd}$ and/or $\mathrm{Cl}$ were used to create patient subgroups. Mean pharmacokinetic data were calculated for each identified sub-group and the sub-groups were compared to verify the existence of a significant difference in pharmacokinetic parameters (ke $\left[\mathrm{h}^{-1}\right], \mathrm{Vd}[\mathrm{L} / \mathrm{kg}]$, and $\left.\mathrm{Cl}[\mathrm{L} / \mathrm{h} / \mathrm{kg}]\right)$ to confirm the validity of the CART-identified breakpoints. An analysis of variance (ANOVA) with Tukey-Kramer Multiple Comparisons Test for data that passed the test for normality or a Kruskal-Wallis Test with Dunn's Multiple Comparison Test for data that did not pass the test for normality (if $>2$ sub-groups were identified) or unpaired t-test (if 2 sub-groups were identified) were used to compare the subgroups (GraphPad InStat version 3.05, 32-bit for Win95/NT; GraphPad Software Inc., La Jolla, California) for differences in pharmacokinetic parameters with a $p<0.05$ considered statistically significant.

Mean PK data of each sub-group were used to explore initial dosing recommendations using first order PK equations for a suggested dose and interval based on inputs for the desired peak and trough concentrations with an infusion time of $1 \mathrm{~h}$. The exploratory gentamicin dose and intervals were subsequently evaluated using Monte Carlo simulation (Oracle Crystal Ball, version 11.1.2.4.000, 32-bit for Windows, Redwood City, California) (MCS). The mean and SDs for $k_{e}, \mathrm{Vd}$, and weight for each determined patient subgroup were input with one million iterations to determine the probability of attaining target steady state peak gentamicin concentrations of $5-10 \mathrm{mg} / \mathrm{L}, 8-12 \mathrm{mg} / \mathrm{L}, 8-15 \mathrm{mg} / \mathrm{L}, 8-20 \mathrm{mg} / \mathrm{L}, 12-$ $20 \mathrm{mg} / \mathrm{L}, 15-20 \mathrm{mg} / \mathrm{L}$ and $>20 \mathrm{mg} / \mathrm{L}$, as well as target trough concentrations of $\leq 2 \mathrm{mg} / \mathrm{L}$ and $\leq 0.5 \mathrm{mg} / \mathrm{L}$ with any given dosing simulation. For the purpose of the MCSs, $\mathrm{k}_{\mathrm{e}}$ and Vd were assigned a lognormal distribution; weight was assumed to have a triangular distribution and was truncated at the value corresponding to the CART analysis breakpoint for weight for the given sub-group. The upper and lower limits for weight selection were truncated at $4 \mathrm{~kg}$ and 0.3 $\mathrm{kg}$, respectively, to reflect values above and below which would be improbable for surviving neonates $(<0.3 \mathrm{~kg})$ and would be greater than 2 SDs from the 
mean of any sub-group weight category. As part of each MCS, an assessment of the probability of attaining a Peak:MIC ratio of $\geq 8$ was completed. The MIC was assumed to have a normal distribution truncated at a minimum of $0.5 \mathrm{mg} / \mathrm{L}$ and maximum of $8 \mathrm{mg} / \mathrm{L}$ (Clinical and Laboratory Standards Institute breakpoint for intermediate susceptibility of Enterobactereaceae to gentamicin [32]) with a mean $\mathrm{MIC}_{90}$ of $2 \mathrm{mg} / \mathrm{L}$ and $\mathrm{SD}$ of $1 \mathrm{mg} / \mathrm{L}$, resembling the current MIC distribution for $E$ coli in Canadian pediatric patients [33].

\section{Results}

\section{Demographics}

Of a total of 99 patients for whom there was documentation of therapeutic drug monitoring (TDM), 60 patients were eligible for study inclusion to complete the pharmacokinetic analysis (Fig. 1 and Table 1). Patients with a rise in $\mathrm{sCr}$ of $>25 \%$ during gentamicin therapy were excluded and represent patients who developed nephrotoxicity while on gentamicin (8/99 patients (8\%)); recognizing that nephrotoxicity may have been multifactorial and no assumptions can be made about causation associated with gentamicin in this retrospective study (Fig. 1).

Forty-five of the 60 neonates (75\%) included in this study were born at $\leq 28$ weeks gestation. The mean ( \pm standard deviation (SD), range) GA of neonates at birth and CGA at gentamicin initiation were $27( \pm 3,23-36)$ weeks and $28( \pm 3,24-36)$ weeks, respectively. Thirty-nine patients $(65 \%)$ had a BW of $<1000 \mathrm{~g}$ (defined as extremely low BW [34]) and 55 patients $(92 \%)$ had a BW of $<1500 \mathrm{~g}$ (defined as very low BW [34]). In this cohort, gentamicin was most commonly used for the treatment of culture negative sepsis $(30 / 60 ; 50 \%)$. Forty-four percent $(16 / 36)$ of all bacterial isolates were gram-negative bacteria (GNB), most commonly Escherichia coli $(7 / 36 ; 19 \%)$ and Klebsiella spp $(5 / 36 ; 14 \%)$ (Table 2).

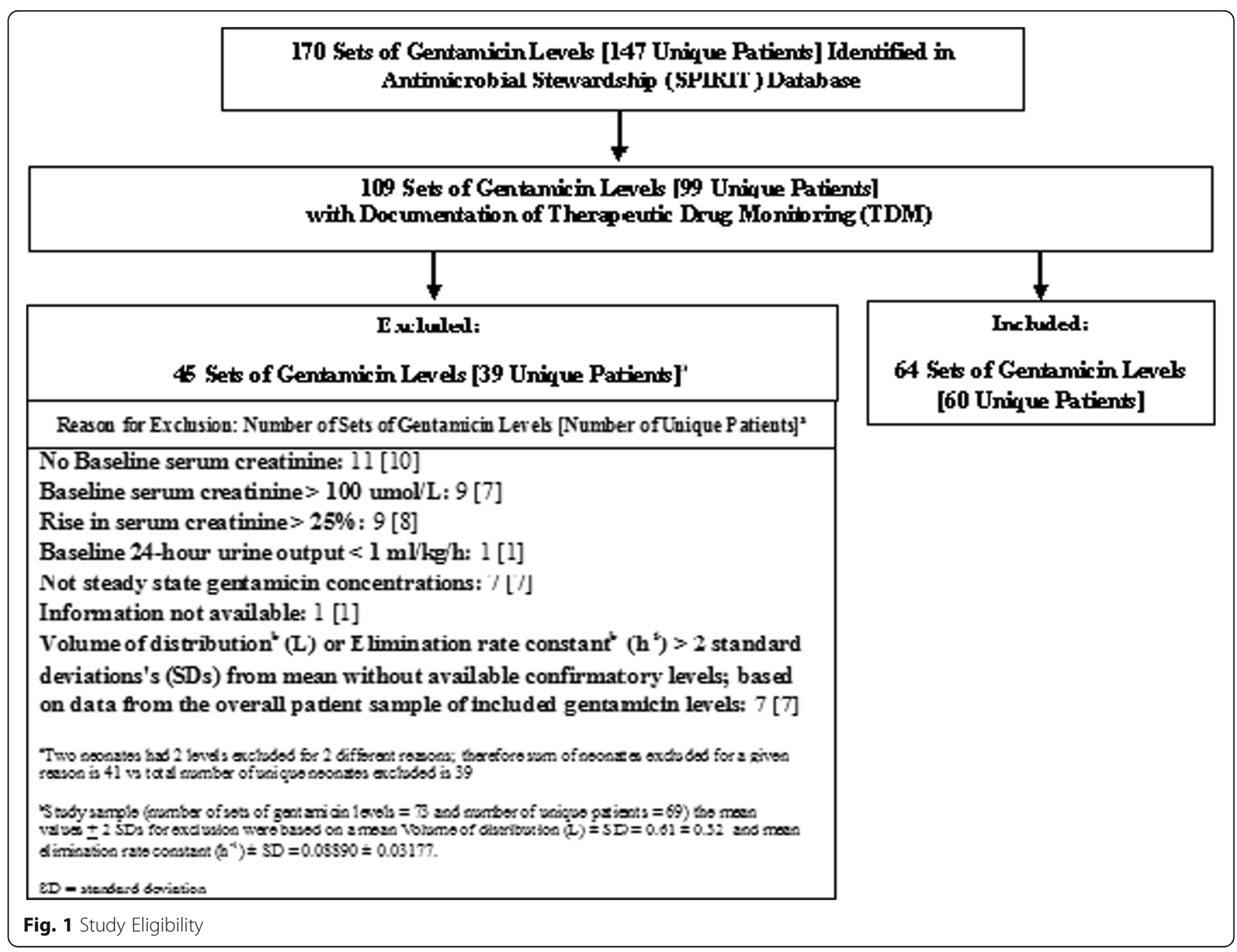


Table 1 Patient Characteristics

\begin{tabular}{|c|c|c|}
\hline Patient Demographics Based on Number of Patients $=60$ & Mean \pm Standard Deviation (Range) & Numbe \\
\hline Gender (Female) & & $24(40)$ \\
\hline Gestational Age at Birth (Weeks) & $27 \pm 3(23-36)$ & \\
\hline Birth Weight (g) & $990 \pm 482(488-2740)$ & \\
\hline Small for Gestational Age Status (Yes) & & $1(2)$ \\
\hline Place of Birth (Outborn) & & $15(25)$ \\
\hline Apgar Score at 1 minute ${ }^{a}$ & $5(1-9)$ & \\
\hline Apgar Score at 5 minute ${ }^{a}$ & $7(2-9)$ & \\
\hline Neonatal Intensive Care Unit Survival & & $56(93)$ \\
\hline \multicolumn{3}{|c|}{ Gentamicin Treatment (Based on Number of Sets of Gentamicin Levels =64) } \\
\hline Post-Natal Age at Gentamicin Initiation (Days) & $10 \pm 12(1-46)$ & \\
\hline Corrected Gestational Age at Gentamicin Initiation (Weeks) & $28 \pm 3(24-36)$ & \\
\hline Weight at Gentamicin Initiation(g) & $1059 \pm 496(488-2789)$ & \\
\hline Gentamicin Dose (mg/kg/dose) & $3.0 \pm 0.7(2-5.6)$ & \\
\hline Gentamicin Dosing Interval (Hours) ${ }^{a}$ & $24(12-36)$ & \\
\hline Duration of Gentamicin Therapy (Days) & $7 \pm 2(2-13)$ & \\
\hline \multicolumn{3}{|c|}{ Indication for Antibiotic Therapy ${ }^{\mathrm{b}}$ (Based on Number of Patients $=60$ ) } \\
\hline Culture Negative Sepsis & & $30(50)$ \\
\hline Necrotizing enterocolitis Septic lleus & & $10(17)$ \\
\hline Respiratory Tract Infection & & $6(10)$ \\
\hline Empiric Treatment ( $<5$ days) & & $5(8)$ \\
\hline Confirmed Sepsis & & $4(7)$ \\
\hline Urinary Tract Infection & & $4(7)$ \\
\hline Meningitis & & $2(3)$ \\
\hline Skin and Soft Tissue Infection & & $1(2)$ \\
\hline Other & & $1(2)$ \\
\hline
\end{tabular}

Laboratory Parameters (Closest to and BEFORE Gentamicin Start Date, unless otherwise noted) (Based on Number of Sets of Gentamicin Levels $=64$ )

Serum creatinine $(\mu \mathrm{mol} / \mathrm{L})$

$$
\begin{aligned}
& 57 \pm 21(24-93) \\
& 64 \pm 23(19-100) \\
& 8 \pm 5(2-24) \\
& 11 \pm 5(3-26) \\
& 4 \pm 1(1-7) \\
& 3 \pm 2(1-19) \\
& 1.1 \pm 0.6(0.2-3.9) \\
& 7.1 \pm 2.2(3.7-17.1)
\end{aligned}
$$$$
\text { Maximum serum creatinine during Gentamicin ( } \mu \mathrm{mol} / \mathrm{L})
$$$$
\text { Blood Urea Nitrogen (mmol/L) }
$$$$
\text { 24-h urine output ( } \mathrm{ml} / \mathrm{kg} / \mathrm{hr} \text { ) }
$$$$
\text { Lowest 24-h urine output during Gentamicin (ml/kg/hr) }
$$$$
\text { Trough gentamicin concentration }(\mathrm{mg} / \mathrm{L})^{\mathrm{c}}
$$$$
\text { Peak gentamicin concentration }(\mathrm{mg} / \mathrm{L})^{c}
$$
Number (\%)

(8)

$4(7)$

$4(7)$

$2(3)$

$1(2)$

$1(2)$

Nephrotoxins \& Ototoxins ${ }^{d}$ (Based on Number of Sets of Gentamicin Levels $=64$ )

Concomitant Nephrotoxins during a course of gentamicin

Concomitant Ototoxins during a course of gentamicin

Vancomycin ${ }^{\mathrm{e}, \mathrm{f}}$

Indomethacin ${ }^{\mathrm{e}, \mathrm{f}}$

Furosemide ${ }^{e, f}$

Amphotericin $B^{\mathrm{e}}$ 
Table 1 Patient Characteristics (Continued)

\begin{tabular}{|c|c|c|}
\hline Patient Demographics Based on Number of Patients $=60$ & Mean \pm Standard Deviation (Range) & Number (\%) \\
\hline Ibuprofen ${ }^{\mathrm{e} f}$ & & $1(2)$ \\
\hline Erythromycin $^{f}$ & & $2(3)$ \\
\hline Prior course of Gentamicin & & $30(47)$ \\
\hline
\end{tabular}

${ }^{a}$ Median reported since apgar scores are ordinal data and standard dosing gentamicin intervals were used (e.g. every 12, 24, or $36 \mathrm{~h}$ ), therefore, gentamicin dosing interval data are ordinal

${ }^{\mathrm{b}}$ Three patients with 2 sets of gentamicin levels had a different diagnosis for each set of gentamicin levels. Therefore, the sum (\%) of total indications is greater than $60(100 \%)$ (i.e. $63(105 \%))$

${ }^{\mathrm{C}}$ Extrapolated concentration using first order pharmacokinetics

${ }^{\mathrm{d} E a c h}$ course of gentamicin may have had greater than one nephrotoxin or ototoxin, therefore, sum of individual nephrotoxins and ototoxins is greater than the total number of courses of gentamicin with a concomitant nephrotoxin or ototoxin

eNephrotoxin

fOtotoxin

\section{Bivariate and multivariable analyses}

Significant predictors $(p<0.05)$ of gentamicin Vd (L) and $\mathrm{Cl}(\mathrm{L} / \mathrm{h})$ from the bivariate screen and multivariable model are detailed in Table 3 . The only covariate that remained significant following MLR for Vd (L) was weight at gentamicin initiation $(P<0.0001)$. Covariates that remained significant following MLR for $\mathrm{Cl}(\mathrm{L} / \mathrm{h})$ were PNA at gentamicin initiation $(p=$ $0.0001)$, gender $(p=0.0447)$, and weight at gentamicin initiation $(p<0.0001)$.

\section{CART analysis}

The optimal CART analyses for $\mathrm{Vd}(\mathrm{L})$ and $\mathrm{Cl}(\mathrm{L} / \mathrm{h})$ produced breakpoints based on the patients' weight at gentamicin initiation, with a forced split at $\leq 850$ $\mathrm{g},>850 \mathrm{~g}-1200 \mathrm{~g}$, and $>1200 \mathrm{~g}$. These breakpoints provided the simplest trees with the lowest relative error (Relative Error for Vd tree $=0.347$; Relative Error for $\mathrm{Cl}$ tree $=0.344$ ). CART identified trees and breakpoints for other parameters in the MLR regression equations (PNA and gender) did not exist.

The mean $\mathrm{k}_{\mathrm{e}}$ and $\mathrm{Cl}(\mathrm{L} / \mathrm{h} / \mathrm{kg})$ for neonates $\leq 850 \mathrm{~g}$ were significantly different from the other weight breakpoints (Table 4). Mean pharmacokinetic parameters for neonates weighing $851-1200 \mathrm{~g}$ versus $>1200 \mathrm{~g}$ were not statistically different $(p>0.05)$ (Table 4$)$. The small number of participants $(n=13$, with 15 gentamicin levels), limited weight range (1210-2789 g; mean $1744 \mathrm{~g}$ ) and wide confidence intervals of the mean calculated pharmacokinetic parameters in the $>1200 \mathrm{~g}$ weight sub-group caused concern regarding the robustness of any dosing recommendations derived for this weight sub-category. As a result, the > $1200 \mathrm{~g}$ weight sub-category of neonates was excluded from further analyses. The significant difference in

Table 2 Bacterial Isolates Cultured at Time of Gentamicin Initiation

\begin{tabular}{|c|c|c|c|c|c|c|c|}
\hline \multicolumn{2}{|c|}{ Number of gentamicin treatment courses } & \multicolumn{6}{|c|}{64 (4 patients had 2 separate gentamicin treatment courses) } \\
\hline \multicolumn{2}{|c|}{ Number of gentamicin treatment courses with a positive culture (\%) } & \multicolumn{6}{|c|}{$24(37.5)$} \\
\hline \multicolumn{2}{|c|}{$\begin{array}{l}\text { Number of gentamicin treatment courses that were Polymicrobial (2 or more } \\
\text { bacterial isolates) (\%) }\end{array}$} & \multicolumn{6}{|c|}{$7(10.9)$} \\
\hline & \multirow[t]{2}{*}{ Total Number of Isolates $n=36(\%)^{\mathrm{a}}$} & \multicolumn{6}{|c|}{ Source of Culture } \\
\hline & & Blood & Cerebrospinal Fluid & Endotracheal Tube & Urine & Eye & Skin \\
\hline Escherichia coli & $7(19)$ & $4(11)$ & $1(3)$ & $1(3)$ & $1(3)$ & 0 & 0 \\
\hline Klebsiella species & $5(14)$ & $1(3)$ & 0 & $2(6)$ & $2(6)$ & 0 & 0 \\
\hline Pseudomonas species & $2(6)$ & 0 & 0 & $1(3)$ & 0 & $1(3)$ & 0 \\
\hline Enterobacter species & $1(3)$ & 0 & 0 & 0 & 0 & 0 & $1(3)$ \\
\hline Raoultella species & $1(3)$ & 0 & 0 & 0 & 0 & 0 & $1(3)$ \\
\hline Gram-positive organisms ${ }^{\mathrm{b}}$ & $15(42)$ & $7(19)$ & $1(3)$ & $1(3)$ & $3(9)$ & $1(3)$ & $2(6)$ \\
\hline Other ${ }^{c}$ & $5(14)$ & 0 & 0 & $5(14)$ & 0 & 0 & 0 \\
\hline
\end{tabular}

\footnotetext{
${ }^{a}$ All percentages are determined from total isolates $(n=36)$
}

b Total of $15 \mathrm{~g}$ positive organisms include coagulase-negative Staphylococcus (12); Enterococcus species (1); Staphylococcus aureus (1) Group B Streptococcus (1)

'Total of 5 'Other' organisms include Mycoplasma spp (1) and Ureaplasma urealyticum (4) 
Table 3 Bivariate and Multivariable Analysis

\begin{tabular}{|c|c|c|c|c|}
\hline \multirow[t]{2}{*}{ Parameter $^{a}$} & \multicolumn{2}{|l|}{ Clearance (L/h) } & \multicolumn{2}{|c|}{ Volume of Distribution (L) } \\
\hline & Bivariate $p$-value & $\begin{array}{l}\text { Multivariable } \\
p \text {-value }\end{array}$ & Bivariate $p$-value & $\overline{\text { Multivariable } p \text {-value }}$ \\
\hline Post-natal age (Days) at gentamicin initiation & $<0.0001$ & 0.0001 & 0.0037 & 0.0563 \\
\hline Gender & 0.0311 & 0.0447 & 0.0667 & - \\
\hline Weight at gentamicin initiation (g) & $<0.0001$ & $<0.0001$ & $<0.0001$ & $<0.0001$ \\
\hline Blood urea nitrogen at baseline (mmol/L) & $<0.0001$ & 0.5855 & $<0.0001$ & 0.6643 \\
\hline Serum creatinine at baseline $(\mu \mathrm{mol} / \mathrm{L})$ & $<0.0001$ & 0.0569 & 0.0011 & 0.4553 \\
\hline Concomitant nephrotoxins & 0.0154 & 0.9332 & 0.0279 & 0.8204 \\
\hline
\end{tabular}

${ }^{a}$ Baseline values needed to be reported within 14 days prior to the initiation of gentamicin; if unavailable, first value taken during course of gentamicin was used as a surrogate

Bold data indicates statistically significant $p$-values for a given parameter with either bivariate or multivariable analysis

both $\mathrm{k}_{\mathrm{e}}$ and $\mathrm{Cl}(\mathrm{L} / \mathrm{h} / \mathrm{kg})$ between the $\leq 850 \mathrm{~g}$ and $851-1200 \mathrm{~g}$ sub-groups (Table 4), and absence of CART identified trees and breakpoints for the other MLR equation covariates (PNA and gender) supports the use of the simple weight range breakpoints of $\leq 850 \mathrm{~g}$ and $851-1200 \mathrm{~g}$ as the sub-groups for practical and convenient empiric gentamicin dosing calculations in neonates.

\section{Monte Carlo simulation}

MCS of weight-based dosing regimens were performed for neonates weighing $\leq 850 \mathrm{~g}$ (Table 5) and those weighing between 851 and $1200 \mathrm{~g}$ (Table 6).
The MCS-identified optimal practical dosing regimens for conventional peaks $(5-10 \mathrm{mg} / \mathrm{L})$ and troughs $(\leq 2$ $\mathrm{mg} / \mathrm{L}$ ) were: $3.5 \mathrm{mg} / \mathrm{kg}$ given iv $\mathrm{q} 48 \mathrm{~h}$ in neonates weighing $\leq 850 \mathrm{~g}$ (probability of target peak and trough attainment of 86 and $100 \%$, respectively) and q24h in neonates weighing $851-1200 \mathrm{~g}$ (probability of target peak and trough attainment of 91 and 97\%, respectively). The MCS-identified optimal practical dosing regimens to produce higher peak concentrations of $12-20 \mathrm{mg} / \mathrm{L}$ and undetectable trough concentrations $(\leq 0.5 \mathrm{mg} / \mathrm{L})$ were: $8-9 \mathrm{mg} / \mathrm{kg}$ dose given iv $\mathrm{q} 72 \mathrm{~h}$ in neonates weighing $\leq 850 \mathrm{~g}$ (probability of target peak and trough attainment of $>73$ and $>85 \%$, respectively) and given $\mathrm{q} 48 \mathrm{~h}$ in neonates between 851

Table 4 Mean pharmacokinetic parameters

\begin{tabular}{|c|c|c|c|c|c|c|c|c|c|c|c|c|c|}
\hline & \multirow{2}{*}{\multicolumn{3}{|c|}{ 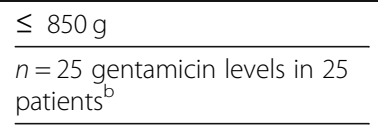 }} & \multirow{2}{*}{\multicolumn{3}{|c|}{$\frac{851-1200 \mathrm{~g}}{n=24 \text { gentamicin levels in } 23_{\text {patients }^{\mathrm{b}}}}$}} & \multirow{2}{*}{\multicolumn{3}{|c|}{$\begin{array}{l}>1200 \mathrm{~g} \\
\begin{array}{l}n=15 \text { gentamicin levels in } 13 \\
\text { patients }\end{array}\end{array}$}} & \multicolumn{4}{|l|}{$p$-value ${ }^{a}$} \\
\hline & & & & & & & & & & \multirow[t]{2}{*}{$\begin{array}{l}\text { Overall } \\
p \text {-value }\end{array}$} & \multirow{2}{*}{$\begin{array}{l}\leq \\
850 \mathrm{~g} \\
\mathrm{Vs} \\
851 \\
- \\
1200 \\
\mathrm{~g}\end{array}$} & \multirow{2}{*}{$\begin{array}{l}\leq \\
850 \mathrm{~g} \\
\text { Vs }> \\
1200 \\
g\end{array}$} & \multirow{2}{*}{$\begin{array}{l}851 \\
- \\
1200 \\
g \\
\text { Vs > } \\
1200 \\
g\end{array}$} \\
\hline & Mean & $\begin{array}{l}95 \% \\
\text { Confidence } \\
\text { Interval }\end{array}$ & Range & Mean & $\begin{array}{l}95 \% \\
\text { Confidence } \\
\text { Interval }\end{array}$ & Range & Mean & $\begin{array}{l}95 \% \\
\text { Confidence } \\
\text { Interval }\end{array}$ & Range & & & & \\
\hline $\begin{array}{l}\text { Elimination rate } \\
\text { constant }\left(h^{-1}\right)\end{array}$ & 0.06415 & $\begin{array}{l}0.05762- \\
0.07068\end{array}$ & $\begin{array}{l}0.0456- \\
0.1139\end{array}$ & 0.09087 & $\begin{array}{l}0.08447- \\
0.09728\end{array}$ & $\begin{array}{l}0.0652- \\
0.1327\end{array}$ & 0.09734 & $\begin{array}{l}0.08519- \\
0.10948\end{array}$ & $\begin{array}{l}0.05693- \\
0.14332\end{array}$ & $\begin{array}{l}< \\
0.0001\end{array}$ & $\begin{array}{l}< \\
0.001\end{array}$ & $\begin{array}{l}< \\
0.001\end{array}$ & $\begin{array}{l}> \\
0.05\end{array}$ \\
\hline Half-life (h) & 10.8 & $9.8-11.8$ & $\begin{array}{l}6.1- \\
15.2\end{array}$ & 7.6 & $7.1-8.2$ & $\begin{array}{l}5.2- \\
10.6\end{array}$ & 7.1 & $6.0-8.2$ & $4.8-12.2$ & - & - & - & - \\
\hline $\begin{array}{l}\text { Volume of } \\
\text { distribution }(L)\end{array}$ & 0.36 & $0.33-0.39$ & $\begin{array}{l}0.22- \\
0.51\end{array}$ & 0.51 & $0.46-0.57$ & $\begin{array}{l}0.33- \\
0.87\end{array}$ & 0.88 & $0.76-1.00$ & $\begin{array}{l}0.53- \\
1.23\end{array}$ & & & & - \\
\hline $\begin{array}{l}\text { Volume of } \\
\text { distribution (L/ } \\
\mathrm{kg} \text { ) }\end{array}$ & 0.55 & $0.50-0.60$ & $\begin{array}{l}0.35- \\
0.83\end{array}$ & 0.50 & $0.46-0.54$ & $\begin{array}{l}0.38- \\
0.76\end{array}$ & 0.52 & $0.43-0.61$ & $\begin{array}{l}0.26- \\
0.96\end{array}$ & 0.2471 & - & - & - \\
\hline Clearance (L/h) & 0.023 & $0.021-0.025$ & $\begin{array}{l}0.016- \\
0.038\end{array}$ & 0.047 & $0.041-0.053$ & $\begin{array}{l}0.028- \\
0.086\end{array}$ & 0.086 & $0.070-0.101$ & $\begin{array}{l}0.040- \\
0.137\end{array}$ & - & - & - & - \\
\hline $\begin{array}{l}\text { Clearance (L/h/ } \\
\mathrm{kg})\end{array}$ & 0.035 & $0.032-0.038$ & $\begin{array}{l}0.026- \\
0.056\end{array}$ & 0.045 & $0.041-0.049$ & $\begin{array}{l}0.032- \\
0.067\end{array}$ & 0.050 & $0.043-0.058$ & $\begin{array}{l}0.028- \\
0.081\end{array}$ & $\begin{array}{l}< \\
0.0001\end{array}$ & $\begin{array}{l}< \\
0.01\end{array}$ & $\begin{array}{l}< \\
0.001\end{array}$ & $\begin{array}{l}> \\
0.05\end{array}$ \\
\hline
\end{tabular}

Multiple Comparison Test for data that did not pass the test for normality

${ }^{a}$ ANOVA with Tukey-Kramer Multiple Comparisons Test for data that passed the test for normality or a Kruskal-Wallis Test with Dunn's Multiple Comparison Test for data that did not pass the test for normality

${ }^{b}$ One patient contributed 1 set of gentamicin levels to weight categories $\leq 850 \mathrm{~g}$ and $851-1200 \mathrm{~g}$

Bold data indicates statistically significant $p$-values for a given parameter with either bivariate or multivariable analysis 
Table 5 Monte Carlo Simulation Results for Neonates Weighing $\leq 850 \mathrm{~g}$

\begin{tabular}{|c|c|c|c|c|c|c|c|c|}
\hline \multicolumn{2}{|c|}{ Dosing Regimen } & \multicolumn{4}{|c|}{$\begin{array}{l}\text { Target Peak Serum Concentration } \\
(\mathrm{mg} / \mathrm{L})\end{array}$} & \multicolumn{2}{|c|}{$\begin{array}{l}\text { Target Trough Serum } \\
\text { Concentration (mg/L) }\end{array}$} & \multirow{2}{*}{$\begin{array}{l}\text { Peak:Minimum Inhibitory Concentration Ratic } \\
\geq 8\end{array}$} \\
\hline Dose (mg/kg) & Dosing Interval (h) & $5-10$ & $12-20$ & $15-20$ & $\geq 20$ & $\leq 2$ & $\leq 0.5$ & \\
\hline $2.5^{\mathrm{a}}$ & 24 & $68.76 \%$ & $0.12 \%$ & $0.01 \%$ & $0.00 \%$ & $81.16 \%$ & $5.18 \%$ & $4.21 \%$ \\
\hline $3.5^{\mathrm{a}}$ & 24 & $80.45 \%$ & $4.61 \%$ & $0.51 \%$ & $0.01 \%$ & $58.24 \%$ & $1.68 \%$ & $11.26 \%$ \\
\hline 3.0 & 48 & $69.05 \%$ & $0.02 \%$ & $0.00 \%$ & $0.00 \%$ & $99.89 \%$ & $78.65 \%$ & $3.26 \%$ \\
\hline $3.5^{\mathrm{b}}$ & 48 & $86.31 \%$ & $0.27 \%$ & $0.00 \%$ & $0.00 \%$ & $99.74 \%$ & $71.89 \%$ & $6.35 \%$ \\
\hline 4.0 & 48 & $87.81 \%$ & $1.53 \%$ & $0.07 \%$ & $0.00 \%$ & $99.45 \%$ & $65.51 \%$ & $9.30 \%$ \\
\hline 4.5 & 48 & $78.22 \%$ & $5.12 \%$ & $0.40 \%$ & $0.00 \%$ & $99.01 \%$ & $59.83 \%$ & $12.54 \%$ \\
\hline 5.0 & 48 & $62.65 \%$ & $12.44 \%$ & $1.53 \%$ & $0.02 \%$ & $98.42 \%$ & $53.62 \%$ & $16.18 \%$ \\
\hline 5.5 & 48 & $45.62 \%$ & $23.47 \%$ & $4.10 \%$ & $0.12 \%$ & $97.69 \%$ & $49.95 \%$ & $20.08 \%$ \\
\hline 6.0 & 48 & $30.54 \%$ & $36.89 \%$ & $8.66 \%$ & $0.42 \%$ & $96.76 \%$ & $45.74 \%$ & $24.21 \%$ \\
\hline 6.5 & 48 & $18.92 \%$ & $50.59 \%$ & $15.39 \%$ & $1.13 \%$ & $95.72 \%$ & $41.94 \%$ & $28.74 \%$ \\
\hline 7.0 & 48 & $11.15 \%$ & $62.11 \%$ & $23.60 \%$ & $2.61 \%$ & $94.51 \%$ & $38.77 \%$ & $33.22 \%$ \\
\hline 7.5 & 48 & $6.10 \%$ & $70.60 \%$ & $32.27 \%$ & $5.19 \%$ & $93.14 \%$ & $35.73 \%$ & $37.96 \%$ \\
\hline 8.0 & 48 & $3.16 \%$ & $74.94 \%$ & $39.79 \%$ & $9.10 \%$ & $91.71 \%$ & $32.96 \%$ & $42.66 \%$ \\
\hline $8.0^{c}$ & 72 & $4.62 \%$ & $73.37 \%$ & $35.31 \%$ & $5.94 \%$ & $99.84 \%$ & $87.65 \%$ & $39.55 \%$ \\
\hline $8.5^{c}$ & 72 & $2.41 \%$ & $76.38 \%$ & $42.25 \%$ & $10.04 \%$ & $99.79 \%$ & $86.31 \%$ & $43.92 \%$ \\
\hline $9.0^{c}$ & 72 & $1.10 \%$ & $75.99 \%$ & $47.24 \%$ & $15.56 \%$ & $99.73 \%$ & $84.88 \%$ & $48.44 \%$ \\
\hline 9.5 & 72 & $0.45 \%$ & $72.46 \%$ & $49.45 \%$ & $22.35 \%$ & $99.65 \%$ & $83.46 \%$ & $52.81 \%$ \\
\hline 10 & 72 & $0.10 \%$ & $66.97 \%$ & $49.27 \%$ & $30.06 \%$ & $99.55 \%$ & $82.07 \%$ & $56.95 \%$ \\
\hline
\end{tabular}

${ }^{\mathrm{a} D o s i n g}$ regimens recommended at Sunnybrook at time of study: $\leq 27$ weeks corrected gestational age (CGA): $2.5 \mathrm{mg} / \mathrm{kg}$ q24h; $28-32 \mathrm{weeks}$ CGA: $3.5 \mathrm{mg} / \mathrm{kg}$ q24h; $33-34$ wks CGA: $4.5 \mathrm{mg} / \mathrm{kg}$ q24h

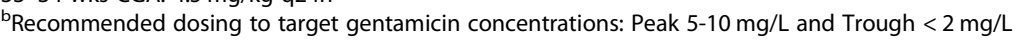

'Recommended dosing to target gentamicin concentrations: Peak $12-20 \mathrm{mg} / \mathrm{L}$ and Trough $\leq 0.5 \mathrm{mg} / \mathrm{L}$

and $1200 \mathrm{~g}$ (probability of target peak and trough attainment of $>75$ and $>84 \%$, respectively).

\section{Discussion}

This retrospective pharmacokinetic study evaluated hospitalized neonates with normal renal function, and a median CGA at gentamicin initiation of $<28$ weeks. Seventy-five percent of those included were born at $\leq$ 28 weeks gestation and $92 \%$ had a BW of $<1500 \mathrm{~g}$. Gentamicin $\mathrm{Cl}(\mathrm{L} / \mathrm{h})$ and $\mathrm{Vd}(\mathrm{L})$ were significantly associated with weight at gentamicin initiation $(\leq 850 \mathrm{~g}$, $851-1200 \mathrm{~g}$, and $>1200 \mathrm{~g}$ ). Since no significant difference in pharmacokinetics existed for neonates weighing $>1200 \mathrm{~g}$ versus $851-1200 \mathrm{~g}$, due to inadequate sample size in the largest weight category, we did not explore the $>1200 \mathrm{~g}$ sub-group further. No CART identified trees with breakpoints for the other MLR equation covariates (PNA and gender) existed. Based on the absence of CART identified trees and breakpoints for PNA and gender and the identification of a significant difference in both $\mathrm{k}_{\mathrm{e}}$ and $\mathrm{Cl}(\mathrm{L} / \mathrm{h} / \mathrm{kg})$ between the $\leq 850 \mathrm{~g}$ and $851-1200 \mathrm{~g}$ sub-groups, the use of the simple weight range breakpoints of $\leq 850 \mathrm{~g}$ and
$851-1200 \mathrm{~g}$ as the sub-groups for practical and convenient empiric gentamicin dosing calculations in neonates is rational. Dosing of $3.5 \mathrm{mg} / \mathrm{kg} / \mathrm{dose}$ administered every $48 \mathrm{~h}$ for neonates weighing $\leq 850 \mathrm{~g}$, and every $24 \mathrm{~h}$ for neonates weighing $851-1200 \mathrm{~g}$ provided the best probability of attaining conventional targets (peak:5-10 mg/L, trough: $\leq 2 \mathrm{mg} / \mathrm{L}$ ). Dosing of $8-9 \mathrm{mg} /$ $\mathrm{kg} /$ dose administered every $72 \mathrm{~h}$ in neonates weighing $\leq 850 \mathrm{~g}$ and every $48 \mathrm{~h}$ in neonates weighing 851-1200 $\mathrm{g}$ provided the best probability of attaining EID targets (peak:12-20 mg/L, trough: $\leq 0.5 \mathrm{mg} / \mathrm{L}$ ).

The strengths of our study include the determination of gentamicin pharmacokinetics in a large sample of premature and low-birth weight neonates for whom data are currently lacking; the identification of significant covariates for $\mathrm{Vd}$ and $\mathrm{Cl}$ with determination of practical weight breakpoints; the utilization of MCS with 1 million iterations to develop simple initial gentamicin dosing nomograms for both conventional and EID for low-birth weight neonates with an excellent probability of target peak and trough attainment; and the provision of tables itemizing probabilities of target attainment (including Peak:MIC 
Table 6 Monte Carlo Simulation Results for Neonates Weighing Between 851 and $1200 \mathrm{~g}$

\begin{tabular}{|c|c|c|c|c|c|c|c|c|}
\hline \multicolumn{2}{|c|}{ Dosing Regimen } & \multicolumn{4}{|c|}{$\begin{array}{l}\text { Target Peak Serum Concentration } \\
(\mathrm{mg} / \mathrm{L})\end{array}$} & \multicolumn{2}{|c|}{$\begin{array}{l}\text { Target Trough Serum } \\
\text { Concentration }(\mathrm{mg} / \mathrm{L})\end{array}$} & \multirow{2}{*}{$\begin{array}{l}\text { Peak: Minimum Inhibitory Concentration Ratio } \\
\geq 8\end{array}$} \\
\hline Dose (mg/kg) & Dosing Interval (h) & $5-10$ & $12-20$ & $15-20$ & $\geq 20$ & $\leq 2$ & $\leq 0.5$ & \\
\hline $2.5^{\mathrm{a}}$ & 24 & $66.21 \%$ & $0.00 \%$ & $0.00 \%$ & $0.00 \%$ & $99.66 \%$ & $27.12 \%$ & $3.27 \%$ \\
\hline $3.5^{\mathrm{a}}$ & 24 & $91.17 \%$ & $0.83 \%$ & $0.02 \%$ & $0.00 \%$ & $96.57 \%$ & $9.94 \%$ & $9.39 \%$ \\
\hline 2.5 & 24 & $66.21 \%$ & $0.00 \%$ & $0.00 \%$ & $0.00 \%$ & $99.66 \%$ & $27.12 \%$ & $3.27 \%$ \\
\hline 3.0 & 24 & $90.28 \%$ & $0.06 \%$ & $0.00 \%$ & $0.00 \%$ & $98.68 \%$ & $16.37 \%$ & $6.08 \%$ \\
\hline $3.5^{\mathrm{b}}$ & 24 & $91.17 \%$ & $0.83 \%$ & $0.02 \%$ & $0.00 \%$ & $96.57 \%$ & $9.94 \%$ & $9.39 \%$ \\
\hline 4.0 & 24 & $77.21 \%$ & $4.42 \%$ & $0.21 \%$ & $0.00 \%$ & $93.13 \%$ & $6.17 \%$ & $13.23 \%$ \\
\hline 4.5 & 24 & $55.29 \%$ & $13.88 \%$ & $1.23 \%$ & $0.01 \%$ & $88.39 \%$ & $4.01 \%$ & $17.50 \%$ \\
\hline 4.5 & 36 & $72.05 \%$ & $5.81 \%$ & $0.27 \%$ & $0.00 \%$ & $99.96 \%$ & $69.44 \%$ & $14.27 \%$ \\
\hline 5.0 & 36 & $50.61 \%$ & $15.89 \%$ & $1.38 \%$ & $0.00 \%$ & $99.90 \%$ & $62.85 \%$ & $18.27 \%$ \\
\hline 5.5 & 36 & $30.74 \%$ & $31.43 \%$ & $4.51 \%$ & $0.05 \%$ & $99.80 \%$ & $56.50 \%$ & $22.73 \%$ \\
\hline 6.0 & 36 & $16.46 \%$ & $49.27 \%$ & $10.87 \%$ & $0.28 \%$ & $99.66 \%$ & $50.67 \%$ & $27.44 \%$ \\
\hline 6.5 & 36 & $7.96 \%$ & $65.32 \%$ & $20.63 \%$ & $0.96 \%$ & $99.42 \%$ & $45.56 \%$ & $32.25 \%$ \\
\hline 7.0 & 36 & $3.50 \%$ & $76.80 \%$ & $32.38 \%$ & $2.61 \%$ & $99.08 \%$ & $40.71 \%$ & $37.26 \%$ \\
\hline 7.5 & 36 & $1.43 \%$ & $82.50 \%$ & $43.50 \%$ & $5.85 \%$ & $98.62 \%$ & $36.70 \%$ & $42.38 \%$ \\
\hline 8.0 & 36 & $0.56 \%$ & $82.76 \%$ & $52.04 \%$ & $11.15 \%$ & $98.06 \%$ & $32.80 \%$ & $47.42 \%$ \\
\hline 8.5 & 36 & $0.21 \%$ & $78.31 \%$ & $56.09 \%$ & $18.62 \%$ & $97.40 \%$ & $29.56 \%$ & $52.36 \%$ \\
\hline 9.0 & 36 & $0.07 \%$ & $70.46 \%$ & $55.41 \%$ & $28.09 \%$ & $94.61 \%$ & $21.62 \%$ & $66.33 \%$ \\
\hline 7.5 & 48 & $14.52 \%$ & $81.31 \%$ & $39.39 \%$ & $4.12 \%$ & $99.99 \%$ & $89.88 \%$ & $40.26 \%$ \\
\hline $8.0^{c}$ & 48 & $7.95 \%$ & $83.70 \%$ & $49.22 \%$ & $8.35 \%$ & $99.99 \%$ & $88.06 \%$ & $45.22 \%$ \\
\hline $8.5^{c}$ & 48 & $4.07 \%$ & $81.27 \%$ & $55.18 \%$ & $14.72 \%$ & $99.98 \%$ & $86.11 \%$ & $50.20 \%$ \\
\hline $9.0^{c}$ & 48 & $2.00 \%$ & $74.96 \%$ & $56.71 \%$ & $23.04 \%$ & $99.96 \%$ & $84.10 \%$ & $54.91 \%$ \\
\hline 9.5 & 48 & $0.94 \%$ & $66.18 \%$ & $54.11 \%$ & $32.88 \%$ & $99.95 \%$ & $82.15 \%$ & $59.48 \%$ \\
\hline 10 & 48 & $0.43 \%$ & $56.10 \%$ & $48.53 \%$ & $43.50 \%$ & $99.93 \%$ & $80.12 \%$ & $63.87 \%$ \\
\hline
\end{tabular}

a Dosing regimens recommended at Sunnybrook at time of study: $\leq 27$ weeks corrected gestational age (CGA): $2.5 \mathrm{mg} / \mathrm{kg}$ q24h; $28-32 \mathrm{weeks}$ CGA: $3.5 \mathrm{mg} / \mathrm{kg}$ q24h; 33-34 wks CGA: $4.5 \mathrm{mg} / \mathrm{kg}$ q24h

${ }^{\mathrm{b}}$ Recommended dosing to target gentamicin concentrations: Peak 5-10 mg/L and Trough $<2 \mathrm{mg} / \mathrm{L}$

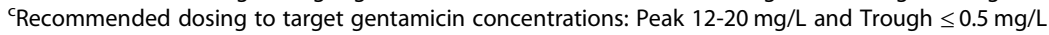

ratio) for a range of potential dosing options enabling institutional selection of initial dosing guidelines based on their GNB susceptibility patterns and desired target serum concentrations. In addition, our rigorous study design which limited the inclusion of gentamicin levels to those with a confirmed time for dose administration and serum sampling increases the validity of our results.

The weaknesses of our study include its retrospective design and associated risk of unrecognized confounders; the inability to generalize our results to neonates $>1200$ $\mathrm{g}$ and SGA infants; and the risk of incomplete gentamicin distribution at time of sampling for peak concentrations. However, since our mean pharmacokinetic parameters were comparable to those reported in other studies [15], our sampling practice is unlikely to have affected the validity of our results.
Similar to other pharmacokinetic studies, our multivariable analysis indicated that the $\mathrm{Vd}$ of gentamicin in neonates is associated with body weight $[15,18-20,28,29]$. Pharmacokinetic studies have identified that extracellular fluid volume correlates closely with bodyweight [35].

Our multivariable analysis indicated that gentamicin clearance in neonates is associated with PNA, as well as bodyweight, and gender. The correlation between PNA and gentamicin elimination has been previously reported in the literature $[19,28,29]$, and is explained by the maturation of renal function in neonates. Since glomerulogenesis proceeds until 32-34 weeks gestation, preterm neonates are expected to have a reduced rate of glomerular filtration compared to their mature counterparts [36]. In the first $48-72 \mathrm{~h}$ of life there is a marked increase in glomerular filtration rate of full term newborns to rates of $8-20 \mathrm{ml} /$ 
min, compared with increases in preterm neonates of only $2-3 \mathrm{ml} / \mathrm{min}[35,37]$. The half-life of elimination of gentamicin is therefore expected to decrease with increasing PNA because it is renally eliminated [37], as evidenced in our study. In addition, bodyweight likely serves as a surrogate marker for physiological maturity. Therefore, it is expected that the half-life of elimination of gentamicin decreases as body weight increases. This relationship was demonstrated in our study, as well as in previously published literature $[15,18-20,29]$.

CART analysis confirmed breakpoints for weight at gentamicin initiation for both $\mathrm{Vd}$ and $\mathrm{Cl}$ and demonstrated that neonates had altered $\mathrm{Vd}(\mathrm{L})$ and $\mathrm{Cl}(\mathrm{L} / \mathrm{h})$ based on these weight breakpoints. This allowed the use of the CART derived weight breakpoints $(\leq 850 \mathrm{~g}$ and $851-1200 \mathrm{~g}$ ) to divide our data into homogenous patient sub-groups for practical empiric gentamicin dosing recommendations and provides a new and convenient nomogram for gentamicin dosing (either conventional or EID) with a MCS demonstrated high probability of target attainment. The mean gentamicin Vd $(0.55 \mathrm{~L} / \mathrm{kg}$ and $0.50 \mathrm{~L} / \mathrm{kg}$ for neonates weighing $\leq 850 \mathrm{~g}$ and $851-1200 \mathrm{~g}$, respectively) and $\mathrm{Cl}$ $(0.035 \mathrm{~L} / \mathrm{h} / \mathrm{kg}$ and $0.045 \mathrm{~L} / \mathrm{h} / \mathrm{kg}$, for neonates weighing $\leq 850 \mathrm{~g}$ and $851-1200 \mathrm{~g}$, respectively) identified in this study are comparable to those reported in a study of infants born at less than 28 weeks gestation $(\mathrm{Vd}=$ $0.50 \mathrm{~L} / \mathrm{kg}$ and $\mathrm{Cl}=0.032 \mathrm{~L} / \mathrm{h} / \mathrm{kg}$ ) [15].

Our study confirms previous reports [2, 27, 38] that GNB, particularly $E$ coli, are emerging as the leading cause of systemic infections in neonates. Recent microbiological reports of $E$ coli isolates from Canadian pediatric patients report a mean $\mathrm{MIC}_{90}$ of $2 \mathrm{mg} / \mathrm{L}$ for gentamicin [33]. Therefore, to meet the PK/PD target of a peak: MIC ratio between 8 and 10, peak gentamicin concentrations should range from 16 to $20 \mathrm{mg} / \mathrm{L}$. A single published study approximates these recommendations by targeting a peak concentration of $15-20 \mathrm{mg} / \mathrm{L}$ in neonates [19]. In this study, initial doses of $10 \mathrm{mg} / \mathrm{kg}$ administered at $36 \mathrm{~h}$ intervals were used in term newborns and 12 $\mathrm{mg} / \mathrm{kg}$ doses administered every $48 \mathrm{~h}$ were used in premature neonates (GA 31-38 weeks) [19]. Our MCS derived initial EID recommendations for gentamicin of $8-9 \mathrm{mg} / \mathrm{kg} /$ dose administered every $72 \mathrm{~h}$ in neonates weighing $\leq 850 \mathrm{~g}$ and every $48 \mathrm{~h}$ in neonates weighing $851-1200 \mathrm{~g}$ has $>73 \%$ probability of attaining a peak between 12 and $20 \mathrm{mg} / \mathrm{L}$ and $>84 \%$ probability of attaining a trough of $\leq 0.5 \mathrm{mg} / \mathrm{L}$. Our work is further supported by results from a recent study concluding that a prolonged dosing interval for gentamicin ranging from 36 to $72 \mathrm{~h}$ was appropriate for neonates weighing less than $1000 \mathrm{~g}$ [25]. However, our results provide a new easy to use gentamicin dosing nomogram for both conventional and EID gentamicin with a MCS demonstrated high probability of target attainment, which has not previously been completed for neonates. In all cases the weight based initial dosing recommendations derived in our study provided a better probability of target attainment than the CGA-based gentamicin dosing regimens used at our institution at the time of this study conduct. In 2014 our centre changed its gentamicin dosing practice to adopt the weight based nomogram developed in this study; where EID is now predominantly used for NICU babies. We have received positive feedback about the simplicity, safety and efficacy of the nomogram from our NICU physicians and pharmacists. Plans are underway to evaluate the safety, efficacy and health care personnel workload of the weight based nomograms for conventional and EID using a pragmatic study design.

Although the study by Lanao et al [19] was published in 2004, higher peak concentration targets have not been routinely adopted by clinicians. Therefore, we chose to report the probabilities of achieving a range of peak gentamicin concentrations with various dosing regimens because GNB MICs, along with desired target peak concentrations, may vary among hospitals. Our MCS dosing tables may assist clinicians in choosing a gentamicin dosing regimen that would be optimal based on their institutional MIC patterns for relevant GNB, such as E. coli.

\section{Conclusions}

The study contributes new data based gentamicin dosing guidelines for both initial conventional and EID in neonates $\leq 1200 \mathrm{~g}$, a patient population underrepresented in neonatal studies and for whom limited data exists for gentamicin dosing. Our results provide clinicians with practical and simple initial dosing recommendations based on weight at time of gentamicin initiation with a high probability of target peak and trough attainment. Confirmatory gentamicin levels (peak and trough with third dose for conventional therapy and a peak and $8-12 \mathrm{~h}$ post level with the first dose of EID) are recommended to further refine dosing. If more prolonged therapy is needed, then repeat levels are recommended to identify changes in the neonate's gentamicin pharmacokinetics with PNA and weight. The gentamicin levels that were targeted in this study reflect accepted safe and effective levels for gentamicin in neonates $[1,2,6,11-26]$. However, due to the retrospective design of our study, a prospective pharmacokinetic clinical study in neonates $\leq$ $1200 \mathrm{~g}$ is needed to confirm the efficacy and safety of the gentamicin EID nomogram recommendations. 


\section{Appendix 1}

Table 7 Sunnybrook Health Sciences Centre's Dosing Recommendations for Gentamicin in Neonates at Time of Study (2013)

\begin{tabular}{lllll}
\hline Corrected Gestational Age [Weeks] & Dose [mg/kg] & Dosing Interval [Hours] & Administration Technique & Serum Concentration Sampling \\
\hline$<28$ & 2.5 & 24 & $1 \mathrm{~h}$ infusion & Immediately following end of infusion \\
$28-32$ & 3.5 & 24 & $1 \mathrm{~h}$ infusion & Immediately following end of infusion \\
$33-34$ & 4 & 24 & $1 \mathrm{~h}$ infusion & Immediately following end of infusion \\
$35-36$ & 2 & 12 & Intravenous bolus & 30 min following bolus \\
$>36$ & 2.5 & 12 & Intravenous bolus & 30 min following bolus \\
\hline
\end{tabular}

\section{Appendix 2}

\section{First Order Pharmacokinetic Equations:}

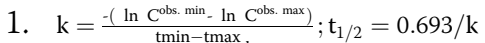

2. $C^{\text {ext. } \max }=\frac{\mathrm{C}^{\mathrm{tm} s} \cdot \max }{\mathrm{e}^{-\mathrm{ktmax}}}$

3. $\mathrm{C}^{\text {ext. } \min }=\mathrm{C}^{\mathrm{e}^{-\mathrm{ktmax}}} \max ^{\mathrm{max}} \mathrm{x} \mathrm{e}^{-\mathrm{k}\left(\mathrm{t}-\mathrm{t}^{\prime}\right)}$

4. $\mathrm{V}=\frac{\text { Dose } \mathrm{x}\left[1-\mathrm{e}^{-\mathrm{kt} \mathrm{t}^{\prime}}\right]}{\mathrm{kt}^{\prime}\left[\mathrm{C}^{\mathrm{ext} . \max }-\mathrm{C}^{\text {ext. } \min } \mathrm{x} \mathrm{e}^{-\mathrm{kt} \mathrm{t}^{\prime}}\right]}$

5. Suggested Dosing Interval:

$$
\mathrm{t}=\frac{-1 / \mathrm{k} \ln \left(C^{\text {des.minss }}\right)+\mathrm{t}^{\prime}}{\left(\mathrm{C}^{\text {des.maxss }}\right)}
$$

Round to 4, 6, 8, 12, 16, 18, 24 etc.

6. Suggested Maintenance Dose:

Dose $=k V C^{\text {des.maxss }} \times \frac{\left(1-\mathrm{e}^{-k \mathrm{t}}\right) \times \mathrm{t}^{\prime}}{\left(1-\mathrm{e}^{-\mathrm{kt}^{\prime}}\right)}$

7. Predicted Peak

$$
C^{\text {pred.maxss }}=\frac{\text { Dose x }\left(1-\mathrm{e}^{-k t^{\prime}}\right)}{t^{\prime} k V x\left(1-e^{-k t}\right)}
$$

\section{Predicted Trough}

$$
\mathrm{C}^{\text {pred.minss }}=\mathrm{C}^{\text {pred.maxss }}\left(\mathrm{e}^{-\mathrm{k}\left(\mathrm{t}-\mathrm{t}^{\prime}\right)}\right)
$$

Key:

$\mathrm{k}=$ elimination rate constant $\left(\mathrm{h}^{-1}\right) ; \mathrm{t}_{1 / 2}=$ half-life $(\mathrm{h})$
Cobs $^{\text {min }} \& \mathrm{C}^{\text {obs.max }}=$ observed minimum and maximum concentrations tmin \& tmax = time post infusion (in hours) of observed minimum and maximum concentrations $C^{\text {ext.min }} \& C^{\text {ext.max }}=$ extrapolated or actual minimum and maximum concentrations $\mathrm{V}=$ volume of distribution $(\mathrm{L}) ; \mathrm{t}=$ dosing interval in hours ; $t^{\prime}=$ infusion time in hours $C^{\text {des.minss }} \& C^{\text {des.maxss }}=$ desired minimum and maximum concentrations at steady state $C^{\text {pred.minss }} \& C^{\text {pred.maxss }}=$ predicted minimum and maximum concentrations at steady state

\section{Abbreviations}

ANOVA: Analysis of variance; BUN: Blood urea nitrogen; BW: Birth weight; CART: Classification and regression tree; CGA: Corrected gestational age; $\mathrm{Cl}$ : Confidence interval; Cl: Clearance; EID: Extended-interval dosing: GA: Gestational age; GNB: Gram negative bacteria; ke: Elimination rate constant; MCS: Monte Carlo Simulation; MIC: Minimum inhibitory concentration; MLR: Multiple linear regression; NICU: Neonatal intensive care unit; PK: Pharmacokinetic; PNA: Post-natal age; sCr: Serum creatinine; SD: Standard deviation; SGA: Small for gestational age; SHSC: Sunnybrook Health Sciences Centre; $t_{1 / 2}$ : Half-life; TDM: Therapeutic drug monitoring; $\vee \mathrm{d}$ : Volume of distribution

\section{Acknowledgements}

Dr. Alex Kiss, Research Design and Biostatistics, Sunnybrook Health Sciences Centre for his assistance with the statistical analysis.

\section{Authors' contributions}

SW conceived the project idea, was the project supervisor and is the senior investigator. SW, MB, ME, DI, CF, WS and EN contributed to the design of the study and manuscript development. SW, MB and ME were responsible for development of the data collection tool. MB, and ME completed the data collection. SW along with MB analyzed the results. All authors read and approved the final manuscript.

\section{Funding}

This research received no specific grant from any funding agency in the public, commercial or not-for-profit sectors.

\section{Availability of data and materials}

The datasets used and/or analysed during the current study are available from the corresponding author on reasonable request.

\section{Ethics approval and consent to participate}

This study was approved by the Sunnybrook Health Sciences Centre (SHSC) Research Ethics Board on February 3, 2014 (Project Identification Number 038-2014), and was conducted in accordance with the Declaration of Helsinki and national and institutional standards. 


\section{Consent for publication}

Not applicable.

\section{Competing interests}

The authors declare that they have no competing interests.

\section{Author details}

'Department of Pharmacy, Sunnybrook Health Sciences Centre, 2075 Bayview Avenue, E-302, Toronto, ON M4N 3M5, Canada. '2Leslie L. Dan Faculty of Pharmacy, University of Toronto, Toronto, ON, Canada. ${ }^{3}$ Division of Infectious Diseases, Sunnybrook Health Sciences Centre, Toronto, ON, Canada. ${ }^{4}$ Sunnybrook Health Sciences Centre Research Institute, Toronto, ON, Canada. ${ }^{5}$ Women and Babies Program, Sunnybrook Health Sciences Centre, Toronto, ON, Canada. ' ${ }^{6}$ epartment of Pharmacy, Hospital for Sick Children, Toronto, ON, Canada. ${ }^{7}$ Department of Paediatrics, University of Toronto, Toronto, ON, Canada. ${ }^{8}$ Present Address: Grandview Medical Centre Family Health Team, 167 Hespeler Rd, Cambridge, ON N1R 3H7, Canada.

Received: 16 April 2019 Accepted: 19 August 2019

Published online: 06 September 2019

\section{References}

1. Contopoulos-loannidis DG, Giotis ND, Baliatsa DV, loannidis JP. Extended -interval aminoglycoside administration for children: a meta-analysis. Pediatrics. 2004;114(1):e111-8. https://doi.org/10.1542/peds.114.1.e111.

2. Touw DJ, Westerman EM, Sprij AJ. Therapeutic drug monitoring of aminoglycosides in neonates. Clin Pharmacokinet. 2009;48(2):71-88. https:// doi.org/10.2165/0003088-200948020-00001.

3. Moore RD, Lietman PS, Smith CR. Clinical response to aminoglycoside therapy: importance of the ratio of peak concentration to minimal inhibitory concentration. J Infect Dis. 1987;155(1):93-9. https://doi.org/10.1 093/infdis/155.1.93.

4. Begg EJ, Peddie BA, Chambers ST, Boswell DR. Comparison of gentamicin dosing regimens using an in-vitro model. J Antimicrob Chemother. 1992; 29(4):427-33. https://doi.org/10.1093/jac/29.4.427.

5. Nicolau DP, Freeman CD, Belliveau PP, Nightingale CH, Ross JW, Quintiliani R. Experience with a once-daily aminoglycoside program administered to 2,184 adult patients. Antimicrob Agents Chemother. 1995;39(3):650-5. https://doi.org/10.1128/AAC.39.3.650.

6. Miron D. Once daily dosing of gentamicin in infants and children. Pediatr Infect Dis J. 2001;20(12):1169-73.

7. Bailey TC, Little JR, Littenberg B, Reichley RM, Claiborne DW. A meta-analysis of extended-interval dosing versus multiple daily dosing of aminoglycosides. Clin Infect Dis. 1997;24:786-95. https://doi.org/10.1093/ clinids/24.5.786.

8. Ali MZ, Goetz MB. A meta-analysis of the relative efficacy and toxicity of single daily dosing versus multiple daily dosing of aminoglycosides. Clin Infect Dis. 1997;24:796-809. https://doi.org/10.1093/clinids/24.5.796.

9. Munckhof WJ, Grayson ML, Turnidge JD. A meta-analysis of studies on the safety and efficacy of aminoglycosides given either once daily or as divided doses. J Antimicrob Chemother. 1996;37:645-63. https://doi.org/10.1093/ $\mathrm{jac} / 37.4 .645$.

10. Barza M, loannidis JP, Cappelleri JC, Lau J. Single or multiple daily doses of aminoglycosides: a meta-analysis. BMJ. 1996;312:338-45. https://doi.org/1 0.1136/bmj.312.7027.338

11. Nestaas E, Bangstad HJ, Sandvik L, Wathne KO. Aminoglycoside extended interval dosing in neonates is safe and effective: a meta-analysis. Arch Dis Child Fetal Neonatal Ed. 2005;90(4):F294-300. https://doi.org/10.1136/adc.2 004.056317.

12. Rao SC, Srinivasjois R, Moon K. One dose per day compared to multiple doses per day of gentamicin for treatment of suspected or proven sepsis in neonates. Cochrane Database Syst Rev. 2016;12:CD005091. https://doi.org/1 0.1002/14651858.CD005091.pub4.

13. Skopnik H, Heimann G. Once daily aminoglycoside dosing in full term neonates. Pediatr Infect Dis J. 1995;14(1):71-2.

14. Hayani KC, Hatzopoulos FK, Frank AL, Thummala MR, Hantsch MJ, Schatz BM, John EG, Vidyasagar D. Pharmacokinetics of once-daily dosing of gentamicin in neonates. J Pediatr. 1997;131(1):76-80. https://doi.org/10.1 016/S0022-3476(97)70127-6.

15. DiCenzo R, Forrest A, Slish JC, Cole C, Guillet R. A gentamicin pharmacokinetic population model and once-daily dosing algorithm for neonates. Pharmacotherapy. 2003;23(5):585-91. https://doi.org/10.1592/ phco.23.5.585.32196.

16. Thingvoll ES, Guillet R, Caserta M, Dicenzo R. Observational trial of a 48-hour gentamicin dosing regimen derived from Monte Carlo simulations in infants born at less than 28 weeks' gestation. J Pediatr. 2008;153(4):530-4. https:// doi.org/10.1016/j.jpeds.2008.04.060.

17. Lundergan FS, Glasscock GF, Kim EH, Cohen RS. Once-daily gentamicin dosing in newborn infants. Pediatrics. 1999;103(6):1228-34.

18. Stickland MD, Kirkpatrick CM, Begg EJ, Duffull SB, Oddie SJ, Darlow BA. An extended interval dosing method for gentamicin in neonates. J Antimicrob Chemother. 2001;48(6):887-93. https://doi.org/10.1093/ jac/48.6.887.

19. Lanao JM, Calvo MV, Mesa JA, Martin-Suarez A, Carbajosa MT, Miguelez F, Dominguez-Gil A. Pharmacokinetic basis for the use of extended interval dosage regimens of gentamicin in neonates. J Antimicrob Chemother. 2004;54(1):193-8. https://doi.org/10.1093/jac/dkh261.

20. García B, Barcia E, Pérez F, Molina IT. Population pharmacokinetics of gentamicin in premature newborns. J Antimicrob Chemother. 2006;58(2): 372-9. https://doi.org/10.1093/jac/dkl244.

21. Vervelde ML, Rademaker CM, Krediet TG, Fleer A, van Asten P, van Dijk A. Population pharmacokinetics of gentamicin in preterm neonates: evaluation of a once-daily dosage regimen. Ther Drug Monit. 1999;21(5):514-9.

22. Begg EJ, Vella-Brincat JW, Robertshawe B, McMurtrie MJ, Kirkpatrick CM, Darlow B. Eight years' experience of an extended-interval dosing protocol for gentamicin in neonates. J Antimicrob Chemother. 2009;63(5):1043-9. https://doi.org/10.1093/jac/dkp073.

23. Hansen A, Forbes P, Arnold A, O'Rourke E. Once-daily gentamicin dosing for the preterm and the term newborn: proposal for a simple regimen that achieves target levels. J Perinatol. 2003;23(8):635-9. https://doi.org/10.1038/sj.jp.7210996.

24. Hoff DS, Wilcox RA, Tollefson LM, Lipnik PG, Commers AR, Liu M. Pharmacokinetic outcomes of a simplified, weight-based, extended interval gentamicin dosing protocol in critically ill neonates. Pharmacotherapy. 2009; 29(11):1297-305. https://doi.org/10.1592/phco.29.11.1297.

25. Valitalo PAJ, van den Anker JN, Allegaert K, de Cock RFW, de Hoog M, Simons SHP, Mouton JW, Knibbe CAJ. Novel model-based dosing guidelines for gentamicin and tobramycin in preterm and term neonates. J Antimicrob Chemother. 2015;70(7):2074-7. https://doi.org/10.1093/jac/dkv052.

26. Alshaikh B, Dersch-Mills D, Taylor R, Akierman AR, Yusuf K. Extended interval dosing of gentamicin in premature neonates $\leq 28$-week gestation. Acta Paediatr. 2012;101:1134-9.

27. Shah P, Lee S, Yoon E, Param Y, Members of the Annual Report Review Committee. Canadian Neonatal Network (CNN). The Canadian Neonatal Network Annual Report 2012. Available at: http://www. canadianneonatalnetwork.org/Portal. Accessed 25 May 2018.

28. Nielsen El, Sandström M, Honoré PH, Ewald U, Friberg LE. Developmental pharmacokinetics of gentamicin in preterm and term neonates: population modelling of a prospective study. Clin Pharmacokinet. 2009;48(4):253-63. https://doi.org/10.2165/00003088-200948040-00003.

29. Fuchs A, Guidi M, Giannoni E, Werner D, Buclin T, Widmer N, Csajka C. Population pharmacokinetic study of gentamicin in a large cohort of premature and term neonates. Br J Clin Pharmacol. 2014;78(5):1090-101. https://doi.org/10.1111/bcp.12444.

30. Sunnybrook Health Sciences Centre. About Sunnybrook - Facts and Figures. Available at: https://sunnybrook.ca/content/?page=statistics. Accessed 16 May 2018.

31. Elligsen M, Walker SAN, Simor A, Daneman N. Prospective audit and feedback of antimicrobial stewardship in critical care: program implementation, experience, and challenges. Can J Hosp Pharm. 2012;65(1):31-6.

32. CLSI. Performance standards for antimicrobial susceptibility testing; 25 th informational supplement. CLSI document M100-S25. Wayne: Clinical and laboratory Standards Institute; 2015.

33. Adam HJ, Baxter MR, Davidson RJ, Rubinstein E, Fanella S, Karlowsky JA, Lagace-Wiens PRS, Hoban DJ, Zhanel GG. Comparison of pathogens and their antimicrobial resistance patterns in pediatric, adult and elderly patients in Canadian hospitals. J Antimicrob Chemother. 2013;68(Suppl 1):i31-7. https://doi.org/10.1093/jac/dkt024.

34. World Health Organization. International statistical classification of diseases and related health problems, tenth revision, 2nd ed. World Health Organization; 2004.

35. Besunder JB, Reed MD, Blumer JL. Principles of drug biodisposition in the neonate. A critical evaluation of the pharmacokinetic-pharmacodynamic 
interface (part I). Clin Pharmacokinet. 1988;14:189-216. https://doi.org/1 0.2165/00003088-198814040-00001

36. Schmidt IM, Chellakooty M, Boisen KA, Damgaard IN, Mau Kai C, Olgaard K, Main KM. Impaired kidney growth in low-birth-weight children: distinct effects of maturity and weight for gestational age. Kidney Int. 2005;68(2): 731-40. https://doi.org/10.1111/j.1523-1755.2005.00451.x.

37. Besunder JB, Reed MD, Blumer JL. Principles of drug biodisposition in the neonate. A critical evaluation of the pharmacokinetic-pharmacodynamic interface (part II). Clin Pharmacokinet. 1988;14:261-86. https://doi.org/1 0.2165/00003088-198814040-00001.

38. Stoll BJ, Hansen N, Fanaroff AA, Wright LL, Carlo WA, Ehrenkranz RA, Lemons JA, Donovan EF, Stark AR, Tyson JE, Oh W, Bauer CR, Korones SB, Shankaran S, Laptook AR, Stevenson DK, Papile L, Poole WK. Changes in pathogens causing early-onset sepsis in very-low-birth-weight infants. N Engl J Med. 2002;347(4):240-7. https://doi.org/10.1056/NEJMoa012657.

\section{Publisher's Note}

Springer Nature remains neutral with regard to jurisdictional claims in published maps and institutional affiliations.

Ready to submit your research? Choose BMC and benefit from:

- fast, convenient online submission

- thorough peer review by experienced researchers in your field

- rapid publication on acceptance

- support for research data, including large and complex data types

- gold Open Access which fosters wider collaboration and increased citations

- maximum visibility for your research: over $100 \mathrm{M}$ website views per year

At BMC, research is always in progress.

Learn more biomedcentral.com/submissions 